\title{
Philosophiques
}

\section{Le corps-machine et le vrai homme}

\section{La controverse entre Arnauld et M. Le Moine, doyen de Vitré}

\section{Aloyse-Raymond Ndiayé}

Volume 24, numéro 2, automne 1997

URI : https://id.erudit.org/iderudit/027448ar

DOI : https://doi.org/10.7202/027448ar

Aller au sommaire du numéro

Éditeur(s)

Société de philosophie du Québec

ISSN

0316-2923 (imprimé)

1492-1391 (numérique)

Découvrir la revue

\section{Citer cet article}

Ndiayé, A.-R. (1997). Le corps-machine et le vrai homme : la controverse entre Arnauld et M. Le Moine, doyen de Vitré. Philosophiques, 24(2), 245-258.

https://doi.org/10.7202/027448ar
Résumé de l'article

Cet article examine les éléments du débat qui oppose, au XVIIe siècle, à propos de la théorie cartésienne du corps-machine, l'abbé Le Moine et Arnauld. Les deux théologiens discutent, plus particulièrement, de l'incompatibilité ou, au contraire, de la compatibilité de cette théorie avec la thèse de l'union substantielle de l'âme et du corps ainsi que des conséquences entraînées par l'application aux êtres animés des lois du mécanisme. La genèse du différend est d'abord rappelée pour ensuite analyser les textes de Descartes qui sont en cause et, enfin, évaluer les arguments respectifs des deux auteurs. 


\title{
LE CORPS-MACHINE EI IF VRAI HOMME La coniroverse enire Arnauld et M. Le Moine, doyen de Vitré
}

\author{
PAR \\ ALOYSE-RAYMOND NDIAYÉ
}

RÉSUMÉ : Cet article examine les éléments du débat qui oppose, au $X V I I^{e}$ siècle, à propos de la théorie cartésienne du corps-machine, l'abbé Le Moine et Arnauld. Les deux théologiens discutent, plus particulièrement, de l'incompatibilité ou, au contraire, de la compatibilité de cette théorie avec la thèse de l'union substantielle de l'âme et du corps ainsi que des conséquences entraînées par l'application aux êtres animés des lois du mécanisme. La genèse du différend est d'abord rappelée pour ensuite analyser les textes de Descartes qui sont en cause et, enfin, évaluer les arguments respectifs des deux auteurs.

ABSTRACT: This article examines the elements of the Seventeenth Century debate about the Cartesian body-machine theory which pitted Abbé Le Moine against Arnauld. Both theologians engaged in specific discussion of the incompatibility or, to the contrary, the compatibility of this theory with the thesis of the substantial union of the body and soul, as well as the consequences of applying mechanical laws to living beings. The origin of the disagreement is first reviewed to then analyse the works of Descartes which are in question and, finally, to evaluate the respective arguments of the two authors.

Dans un écrit intitulé Traité de l'essence du corps et de l'union de l'âme avec le corps, contre la philosophie de M. Descartes, l'auteur, M. Le Moine, doyen du chapitre de Vitré, en Bretagne, doute que Descartes " ait parfaitement connu l'essence et les propriétés des corps vivants, [...] l'excellence du corps humain et sa véritable subordination à l'âme ${ }^{1}$ ». Ce qui est, ici, visé par l'abbé Le Moine, c'est l'application des lois du mécanisme non seulement aux êtres inanimés mais encore aux êtres animés, aux corps vivants ou " organisés ", selon la terminologie aristotélicienne, en particulier

1. Euvres de messire Antoine Arnauld, docteur de la maison et société de Sorbonne, publiées par G. Du Pac De Bellegarde et J. Hautefage, Paris et Lausanne, 1775 $1783,43 \mathrm{~T}$ en $38 \mathrm{vol}$. in $4^{\circ}$. Réimpression anastatique, Bruxelles, 1964. Cité par Arnauld dans Euvres complètes, tome 38. 
au corps humain. Cette théorie du corps-machine qui a pour conséquence l'instrumentalisation du corps ne rend pas compte, selon le doyen de Vitré, de la « communication " entre le corps et l'âme, de leur interaction qui caractérise le vrai homme. C'était suffisant pour provoquer la réaction d'Arnauld qui passe au XVII ${ }^{e}$ siècle pour être le plus ardent défenseur de l'orthodoxie cartésienne. La controverse qu'il engage contre l'abbé Le Moine le conduit une fois de plus à prendre part à un débat de philosophie qui a des incidences théologiques. La théorie du corps-machine est-elle incompatible avec l'union substantielle de l'âme et du corps ? Est-elle contraire à la religion ? C est ce point précis de la discussion, aux aspects multiples, que nous allons privilégier.

Dans un premier temps, nous rappellerons la genèse du différend qui oppose Arnauld et l'abbé Le Moine. Nous analyserons ensuite les textes de Descartes qui inspirent la discussion avant d'exposer les arguments des deux théologiens.

\section{Le contexte historique à Port-Royal}

Arnauld était en Hollande quand il reçut, en 1680, l'écrit de l'abbé Le Moine. C'est la mère Angélique de Saint-Jean qui le lui envoya de Port-Royal. II s'était réfugié en Hollande en compagnie de M. l'abbé de Pontchâteau, pour fuir la persécution de Louis XIV contre Port-Royal. Contraint de se cacher en passant d'une retraite à l'autre, il était obligé de vivre dans l'anonymat. C'est durant cette période mouvementée qu'il reçut le traité anti-cartésien de Le Moine. Il lui fit une réponse intitulée Examen d'un Écrit qui a pour titre : Traité de l'essence du corps et de l'union de l'âme avec le corps contre la philosophie de M. Descartes. Ce texte se trouve au tome 38 de ses Euvres completes ${ }^{2}$. M. l'abbé de Pontchâteau jugea utile de faire lire la réponse d'Arnauld à $M$. de Neercassel, a rchevêque d'Utrecht. Le prélat y consentit avec plaisir et, après en avoir pris connaissance, fit cet aveu à M. l'abbé de Pontchâteau :

Je lis pour la seconde fois ce que (M. Arnauld) a écrit pour la défense des cartésiens. Je le trouve, comme tous ses ouvrages, parfaitement beau. Ce qu'il traite dans la première et la seconde partie est de très grande utilité pour la théologie, et ce qu'il traite dans la quatrième donne grande lumière à ce que les cartésiens enseignent touchant l'union cle l'âme avec le corps. Ne serait-il pas à propos de faire imprimer ce Traité ?

La proposition de l'archevêque d'Utrecht de faire imprimer la réponse d'Arnauld se heurta aux réticences de $\mathrm{M}$. de Saci, celui qui servit de guide spirituel à Pascal et dont les Entretiens nous sont rapportés par Fontaine. M. de Saci qui n'était pas très favorable au

2. Ibid. ; nous résumons ici les commentaires historiques des éditeurs des Euvres complètes d'Arnauld, tome 38 . On pourra consulter avec grand intérêt le beau livre d'Émile Jacques: Les années d'exil d'Antoine Arnauld (1679-1694), Louvain, Nauwelaerts, $1976,812 \mathrm{p}$. 
cartésianisme et moins philosophe qu'Arnauld, a lu avec beaucoup de satisfaction, nous dit Pontchâteau, l'écrit contre l'anti-cartésien. Il précise, par ailleurs, que Saci aurait néanmoins souhaité qu'en défendant la philosophie, Arnauld en parlât en théologien. Ce que regrette Saci, c'est de constater que Arnauld néglige ses œuvres de piété en consacrant trop de temps à la philosophie. Il aurait donc aimé qu'il accordât plus de place à l'instruction et à la piété, a en imitant Saint Augustin qui a établi les grandes vérités de la Grâce, en réfutant les hérétiques qui la combattaient . L'opposition de M. de Saci retarda donc la publication de la réponse d'Arnauld contre l'écrit du doyen de Vitré. Elle parut en même temps que ses Euvres complètes, sous la forme d'une lettre adressée à la mère Angélique de Saint-Jean. Arnauld insérera cependant quelques extraits de sa réponse dans sa lettre à $\mathrm{M}$. Du Vaucel du 19 octobre 1691 et dans la première partie de ses Difficultés à $M$. Steyeart .

Les réserves de M. de Saci sur l'opportunité d'imprimer la réponse d'Arnauld nous apprennent qu'à Port-Royal, il n'y avait pas unanimité sur la conduite à tenir à l'égard de la philosophie. Arnauld faisait figure de chef de file du petit groupe des port-royalistes. Mais, il ne faut pas s'y tromper, dans la communauté, tous n'étaient pas favorables au cartésianisme.

Aussi, les avis se partageaient-ils sur la part qu'il convenait d'accorder aux thèses philosophiques chères à Arnauld. Par contre, ses amis ne cessaient de l'encourager à poursuivre son œuvre théologique, même quand il se trouvait en exil en Hollande. Au moment où il reçut le Traité de M. Le Moine, Arnauld se préparait à s'engager dans une longue polémique avec Malebranche, provoquée par la publication, par l'oratorien, en 1680, du Traité de la Nature et de la Grâce. Il sera encouragé dans cette entreprise par Bossuet, hostile autant que lui aux nouveautés théologiques du père Malebranche. Cette polémique durera trop longtemps au point que Nicole, qui pourtant a collaboré avec Arnauld dans l'Art de penser, en éprouvera de la gêne. La controverse avec l'oratorien portait, en effet, sur la nature des idées. Nicole se montre plutôt déçu et regrette qu'Arnauld ne s'attaque pas directement aux erreurs théologiques de son adversaire. À l'exception de Pontchâteau, qui est son plus proche collaborateur, et de Nicole, malgré quelques réserves, tous les autres opposent une vigoureuse résistance à l'engagement cartésien d'Arnauld. Dans l'opposition, c'est Du Vaucel qui est allé le plus loin. Par ses prises de position, Arnauld pouvait donner l'impression d'engager tout le groupe des port-royalistes. Ce qui n'était pas du goût de tout le monde. Lorsque Jurieu, dans la Politique du clergé, accusa les théologiens de Port-Royal d'être aussi attachés au cartésianisme qu'au christianisme, Arnauld lui fit une réponse dans l'Apologie pour les Catholiques en prenant la défense de ses amis et de Descartes. Sa réplique cependant ne fut pas appréciée par tous, parce qu'il donnait l'impression que son engagement personnel pour le 
cartésianisme était partagé par ses amis. Dans le premier volume de l'Apologie, il précisait, en effet, qu'à l'Oratoire, la philosophie nouvelle n'était pas aussi généralement acceptée que le croyait Jurieu. Mais est-ce à dire qu'elle faisait l'unanimité à Port-Royal ?'En l'absence d'une mise au point, le lecteur pourrait bien en conclure que quelles que soient leur bonne foi et leur volonté de rester fidèles à l'Église, les théologiens de Port-Royal éprouvaient tous un fort attachement pour le cartésianisme. Aussi, Du Vaucel, dans ses Observations sur la philosophie de Descartes, prit-il ouvertement position contre la philosophie cartésienne. Il alla même plus loin. Dans une note latine adjointe à la copie des Observations, il fit le reproche à Arnauld d'avoir laissé entendre que les Messieurs de Port-Royal étaient cartésiens, donnant ainsi implicitement raison à Jurieu.

Comme on le voit, le petit groupe de Port-Royal ne s'est jamais senti engagé, autant qu'Arnauld, ni entièrement concerné par la défense du cartésianisme. C'est parce que les port-royalistes s'étaient donné comme règle de se garder de tout attachement à des opinions humaines dès que la théologie était en cause. Ils auraient donc préféré, c'est le sentiment de Saci, voir Arnauld s'intéresser davantage à la théologie, entendant par là, la théologie positive ou historique, celle des Pères, celle qu'il a su utiliser dans la Fréquente communion, en 1643, et qui lui a valu sa réputation de théologien, de défenseur de l'Église, à travers toute l'Europe. Son intérêt pour la philosophie, particulièrement la philosophie cartésienne, le singularise donc dans son entourage. La philosophie, estime Arnauld, doit intéresser le théologien. Elle mérite qu'il lui consacre de son temps. Mais il faut préciser que lorsque Arnauld s'engage dans le débat philosophique, il reste animé par ses préoccupations pastorales et théologiques. À la mère Angélique de Saint-Jean qui lui a adressé le Traité de l'abbé Le Moine, en lui demandant son sentiment, il avouera qu'il a hésité à le lire de peur qu'il ne le détourne d'autres occupations plus importantes.

Telles sont donc les conditions dans lesquelles a lieu la controverse entre Arnauld et le doyen de Vitré. Nous retiendrons pour notre analyse la question relative à la doctrine de corpsmachine.

\section{Machines naturelles et machines artificielles}

L'essentiel de la critique du doyen de Vitré se résume dans cette déclaration que nous rapporte Arnauld :

Je veux que M. Descartes, comme grand Mathématicien, ait parfaitement connu
l'essence et les propriétés des machines, soit naturelles soit artificielles ; mais il
n'a certainement pas connu l'essence et les propriétés des corps vivants. Il n'a pas
connu l'essence du corps humain, et sa véritable subordination à I'àme, en le
faisant passer pour son instrument et une machine de l'âme, et non pour sa partie,
son compagnon, son image vivante; l'internonce de l'âme aux choses de ce
monde, et des choses de ce monde à l'âme, un miroir à deux faces, l'une 
spirituelle, l'autre matérielle qui représente d'un côté les qualités au corps corporellement, et de l'autre les qualités des corps à l'âme spirituellement ; en quoi il a étouffé en lui-même le sentiment et la voix universelle de la nature, qui est la maîtresse des philosophes ${ }^{3}$.

La critique s'adresse à Descartes mathématicien, à l'homme de science, au savant. Se référant aux ceuvres scientifiques de Descartes, il lui reconnaît le mérite d'avoir " parfaitement connu l'essence et les propriétés des machines, soit naturelles soit artificielles $»$. Ses œuvres qui traitent de la question concernent la physique : le Traité du Monde, le Discours de la Méthode, les Principes de la Philosophie. Dans ces différents textes, Descartes considère, en effet, les corps, les choses matérielles comme des machines. Ce sont des machines naturelles comparables aux machines artificielles. Le doyen de Vitré ne trouve pas à redire contre l'assimilation que fait Descartes des choses matérielles à la machine qui résulte de l'explication mécaniste et mathématique que la philosophie cartésienne nous donne du monde. Examinons d'un peu plus près ces textes cartésiens.

Au chapitre VI du Traité du Monde, Descartes nous invite à " sortir hors de ce Monde pour en venir voir un autre tout nouveau n, qu' il fait naître devant nous, dans “ les espaces imaginaires ». Ce nouveau monde est aussi l'œuvre de Dieu auquel il fait appel dans cette fiction : "Supposons que Dieu crée de nouveau tout autour de nous tant de matière que, de quelque côté que notre imagination se puisse étendre, elle n'y aperçoive plus aucun lieu qui soit vide ${ }^{4}$ n. La matière ainsi créée occupe tout l'espace. Elle est assimilée à l'étendue divisible et susceptible d'être mue. La suite du texte nous fait voir que dans cette masse informe, Dieu introduit le mouvement qui sera ainsi la cause de la diversité des êtres :

Supposons que Dieu la divise véritablement en plusieurs telles parties [...] que toute la clistinction qu'il y met consiste dans la diversité des mouvements qu'il leur donne, faisant que dès le premier instant qu'elles sont créées, les unes commencent à se mouvoir d'un côté, les autres d'un autre; les unes plus vite, les autres plus lentement (ou même si vous voulez, point du tout) et qu'elles continuent par après leur mouvement suivant les lois ordinaires de la Nature. Car Dieu a si merveilleusement établi ces lois qu'encore que nous supposions qu 'il ne crée rien de plus que ce que j'ai dit et même qu'il ne mette en ceci aucun ordre ni proportion, mais qu'il en compose un chaos le plus confus [...] elles sont suffisantes pour faire que les parties de ce chaos se démêlent d'elles-mêmes et se disposent en si bon ordre qu'elles auront la forme d'un Monde très parfait et dans lequel on pourra voir non seulement de la lumière, mais aussi toutes les autres choses, tant générales que particulières, qui paraissent dans ce vrai Monde.

Tout se passe comme si les lois de la Nature font tout, une fois donnée par Dieu la première impulsion de mouvement.

3. Cité par Arnauld dans Examen du Traité de l'essence du corps, O.C., tome 38, p. 61.

4. Descartes, Traité du Monde dans Euvres philosophiques, Paris, Garnier, tome 1, 1963, p. 344 puis p. $346-347$. 
Le chapitre VII du Traité expose les lois de la Nature qui ont été établies par Dieu. Elles suffisent à elles seules à veiller à l'organisation, à la conservation et au développement du monde. Le monde que Descartes fait naître devant nous ne reconnaît qu'une seule causalité mécanique pour expliquer les phénomènes qui s'y déroulent. Il exclut toutes considérations finalistes et qualitatives. Ce monde " feint " de Descartes est entièrement mathématique. L'étendue en constitue l'essence, une étendue géométrique et indéfinie. Bien que Descartes nous le présente comme une "fable ", tout ce que nous y observons est tout semblable à ce que nous voyons dans le vrai monde.

Ainsi donc, à partir de son Monde " feint „, Descartes retrouve le vrai monde, celui dans lequel nous vivons, celui de nos expériences. Le projet de Descartes est, en effet, de partir des principes qu'il a posés dans son Monde, c'est à dire des principes d'une physique purement mécaniste, pour retrouver le réel dans toute sa richesse, sa diversité et qui constitue le contenu de notre expérience. Ces principes, quels sont-ils? La matière assimilée à l'étendue, le mouvement et les lois du mouvement qui ne sont rien d'autre que les lois de la Nature qui sont déduites de manière a priori. Une fois en possession de ces principes, Descartes se livre, dans ses différents textes consacrés à la physique, à la description des phénomènes de ce monde visible. " J'ai décrit ", dit-il, " cette Terre et généralement tout le monde visible comme si c'était une machine en laquelle il n'y eut rien du tout à considérer que les figures et les mouvements de ses parties $^{5}$ ». Dans ses descriptions il est question des planètes, de la lumière, des montagnes, des mers, des fontaines, des rivières, des mines, des plantes... Tous ces corps naturels sont étudiés comme des machines, c'est-à-dire par la simple considération de leurs figures, mouvements, grandeurs. Ces notions sont claires. Ce sont des principes de la géométrie et des mécaniques. Ces mêmes principes s'appliquent aux machines artificielles que sont " les corps composés par l'artifice des hommes ». Descartes ne trouve a aucune différence entre les machines que font les artisans et les divers corps que la nature seule compose, sinon que les effets des machines ne dépendent que de l'agencement de certains tuyaux, ou ressorts, ou autres instruments, qui, devant avoir quelque proportion avec les mains de ceux qui les font, sont toujours si grands que leurs figures et mouvements se peuvent voir, au lieu que les tuyaux et ressorts qui causent les effets des corps naturels sont ordinairement trop petits pour être aperçus de nos sens ${ }^{6}$. Toutes les parties visibles ou invisibles de la matière ont la même essence et les mêmes propriétés. De même, toutes les machines artificielles du fait qu'elles obéissent aux règles de la mécanique sont naturelles. Il y a donc une unité de la nature garantie par l'universalité des règles de la mécanique. En

5. Descartes, Les Principes de la philosophie, IV, art. 188, Garnier, tome 3, p. 503.

6. Ibid, art. 203, p. 219.220. 
d'autres termes, pour prendre un exemple, il est tout aussi naturel à un arbre de produire ses fruits qu'à une montre d'indiquer l'heure, bien qu'elle soit une machine artificielle. Dans un cas comme dans l'autre, ce sont les mêmes règles de la mécanique, donc de la physique, qui président aux effets qui résultent de leur fonctionnement.

Il est manifeste que la physique de Descartes innove par rapport à la scolastique, parce qu' elle ne retient comme principes d'explication que des notions claires et distinctes : la figure, le mouvement, la grandeur qui relèvent de la géométrie et de la mécanique. Le doyen de Vitré est bien informé de cet aspect de la physique de Descartes puisqu'il admet, sans équivoque, qu'il a a parfaitement connu l'essence et les propriétés des machines soit naturelles soit artificielles ", c'est-à-dire des corps matériels et inanimés. Informé des textes de Descartes comme le prouve son jugement, le doyen de Vitré a bien vu que la doctrine cartésienne du corps-machine va plus loin. Il ne trouve pas à redire contre cette doctrine tant qu'elle ne concerne que les choses matérielles. Mais Descartes estime que ce qui vaut pour les êtres matériels, inanimés, vaut aussi pour les corps vivants, considérés comme des machines très perfectionnées. Sur ce point, l'abbé Le Moine ne suit pas Descartes. Il lui reproche au contraire d'avoir ignoré l'essence et les propriétés des corps vivants. Il n'a pas connu l'excellence du corps humain a et sa véritable subordination à l'âme, en le faisant passer pour son instrument et une machine de l'âme et non pour sa partie, son compagnon, son image vivante ; l'internonce de l'âme aux choses de ce monde, et des choses de ce monde à l'âme, un miroir à deux faces, l'une spirituelle, l'autre matérielle qui représente d'un côté les qualités aux corps corporellement, et de l'autre les qualités des corps à l'âme spirituellement ; en quoi il a étouffé en lui-même le sentiment et la voix universelle de la nature qui est la maîtresse des philosophes ${ }^{7}$ ». Cela revient à dire que le doyen de Vitré accepte les analyses du Traité du Monde et de ceux qui traitent par ailleurs de la physique, mais qu'il rejette celles du Traité de l'homme, que Descartes considère comme une partie du Traité du Monde. Ces deux essais relèvent, selon le philosophe, de la physique. Examinons d'un peu plus près ce que disent les textes pour mieux comprendre les critiques du doyen de Vitré.

Dans le Traité de l'homme, Descartes procède comme dans le Traité du Monde. La théorie de l'homme qu'il nous présente est apparemment une fiction : « Je suppose ", dit-il, " que le corps de l'homme n'est autre chose qu'une statue ou machine de terre que Dieu façonne tout exprès, pour la rendre la plus semblable à nous qu'il est possible ${ }^{8}$. Descartes commence par distinguer l'âme du corps pour les analyser séparément, avant de a nous montrer

7. Voir note 3.

8. Descartes, Traité de l'homme, Garnier, tome 1, p. 378 ss. 
comment ces deux natures doivent être jointes et unies, pour composer des hommes qui nous ressemblent ».

La méthode que suit Descartes consiste donc à partir comme précédemment d'une hypothèse, pour rejoindre le réel vécu. Il étudie une machine, le corps de l'homme que les analyses précédentes du Monde autorisent à expliquer selon les règles de la mécanique. En d'autres termes, pour expliquer les fonctions biologiques du corps de l'homme, du colps vivant, il convient de suivre les règles de la mécanique. C'est supposer dès lors que le fonctionnement du corps humain est comparable $\propto$ aux horloges, $[\ldots]$ fontaines artificielles, $[\ldots]$ moulins et autres semblables machines qui n'étant faites que par des hommes, ne laissent pas d'avoir la force de se mouvoir d'elles-mêmes en plusieurs diverses façons [...]". Descartes ne s'attarde pas à expliquer certains détails. Il nous renvoie à l'étude de l'anatomie classique si nous voulons en savoir plus sur les « diver'ses pièces "qui composent cette machine, c' est-à-dire les os, les muscles, les nerfs... Mais a pour celles qui, à cause de leur petitesse, sont invisibles, je vous les pourrai plus facilement et plus clairement faire connaître, en vous parlant des mouvements qui en dépendent; si bien qu'il est seulement ici besoin que j'explique par ordre ces mouvements, et que je vous dise par même moyen quelles sont celles de nos fonctions qu'ils représentent ».

Il s'agit bien d'expliquer les fonctions biologiques du corps, en montrant par ordre les mouvements qui en résultent et comment ils dépendent les uns des autres. Comme on le voit, cette théorie de l'homme que Descartes nous présente dans le Traité de l'homme est d'inspiration entièrement mécaniste. Il étudie une machine, en décrit le fonctionnement, le mécanisme, et suppose que dans la réalité les choses se passent de la même façon. Il reconnaît que le mécanisme de l'être vivant est analogue au mécanisme physique. Si Descartes procède de la sorte, c'est qu'il a le souci de comprendre. C'est sa préoccupation majeure. Comprendre pour Descartes, c'est voir, c'est avoir l'intuition claire. Pour comprendre le vivant, il passe par l'espace, par l'étendue qui sert d'application aux mathématiques qui ont le privilège de nous assurer la connaissance certaine des choses. D' où la réduction des corps à une machine pour ne retenir que les éléments qui relèvent de la géométrie et de la mécanique, à savoir l'étendue, les mouvements et la grandeur. La philosophie de Descartes rompt ainsi avec la scolastique. Il établit par contre une continuité entre les corps inanimés et les corps animés ou * organisés ». 


\section{Le Corps-machine et le vai homme}

\section{Dieu ne ressuscile pas les machines}

Cest précisément cette continuité que refuse le doyen de Vitré. II trouve indigne de l'homme de considérer son corps comme une machine. C'est, pour lui, la preuve que Descartes n'a pas connu l'excellence du corps humain. Cette dignité, il la tire de son union intime avec l'ârne, union voulue par Dieu. Or, prétendre que notre corps est une machine, c'est supposer que Dieu " ressuscite des machines $^{9}{ }^{\text {}}$. Cela heurte la foi chrétienne. Cet argument du doyen de Vitré a retenu l'attention de Arnauld puisqu'il nous le rapporte dans sa défense de Descartes. Il est d'inspiration profondément thomiste. L'on ne comprendrait pas, en effet, l'immortalité de l'âme, si le corps n'était que machine, c'est-à-dire un corps étranger et extérieur à l'âme, obéissant à des lois qui lui sont propres, celles du mécanisme universel. Une des fonctions essentielles de l'âme est la connaissance. La connaissance du monde passe par le corps, par l'entremise des sens. Séparée du corps, l'âme est en état de violence. Elle a besoin du corps pour connaître. La résurrection des corps lui permet de retrouver son pouvoir de connaître. Le doyen de Vitré a donc bien vu que le concept de machine appliqué à l'homme introduit une relation d'extériorité et instrumentale qui ne correspond pas à la nature de l'âme. D'où le reproche qu'il adresse à Descartes d'avoir fait passer le corps « pour instrument et une machine de l'âme, et non pour sa partie, son compagnon, son image vivante ; l'internonce de l'âme aux choses et des choses de ce monde à l'âme, un miroir à deux faces, l'une spirituelle, l'autre matérielle qui représente d'un côté les qualités aux corps corporellement et de l'autre les qualités des corps à l'âme spirituellement [...] $\mathrm{n}$.

Dans le fond, le doyen de Vitré reste attaché à la conception scolastique. Si le corps est une machine, l'on ne peut plus rendre compte de l'interaction des deux substances, donc de l'union de l'âme et du corps.

En effet, le corps-machine est, par définition, pour la scolastique, un corps inanimé. Les corps animés sont « des corps joints à des âmes spirituelles ou matérielles qui en doivent être réellement distinctes, comme les formes substantielles le sont de la matière qu'elles informent». Les philosophes de l'École ne peuvent pas appeler machine les corps animés. Une machine est nécessairement un objet artificiel, produit par l'homme auquel, par conséquent, ne peut être jointe une âme, puisque l'homme n'a pas le pouvoir de créer des âmes. Les mouvements que nous observons dans ces machines artificielles ne sont que le fait de leurs ressorts. L'âme, au contraire, " communique sa vie et ses propriétés à son corps par une

9. Cité par Arnauld dans Examen du traité de l'essence du corps, O.C., tome 38, p. 152. 
communication réelle, formelle et substantielle ". Son corps ne peut donc pas être assimilé, en aucune façon, à une machine.

La critique du doyen de Vitré, par sa virulence, ne peut se comprendre que parce qu'il se réfère aux textes scientifiques de Descartes où se trouve exposée sa théorie de l'homme. Le Traité de l'homme a pu le décevoir dans la mesure où, d'entrée de jeu, après avoir annoncé que l'explication complète de l'homme suppose la solution du problème de l'union de l'âme et du corps, Descartes n'aborde pas le problème dans la suite de ses analyses. L'union reste une supposition, mais elle n'est point démontrée. Elle le sera dans la sixième des Méditations métaphysiques. C'est ce qui explique la remarque du doyen de Vitré que nous rapporte fidèlement Arnauld dans sa réplique : « Si M. Descartes a trouvé quelque nouveau secret dans la nature, c'est celui d'avoir séparé les âmes des corps plutôt que de les unir ensemble [...] il a trouvé plutôt l'art de séparer l'âme du corps que le mystère de leur union naturelle ${ }^{10}$ ". Ce qui implicitement voudrait dire que Descartes ne nous donne pas la théorie du vrai homme. La question de l'immortalité de l'âme n'a de sens que pour' qui définit le vrai homme composé de l'âme et du corps.

\section{Le vrai homme}

Qu'en pense Arnauld? Il écarte ce qu'il considère comme " inintelligible " et "énigmatique " : l'idée que le corps est comme un miroir à deux faces, l'une spirituelle, l'autre matérielle. Ce langage est a mystérieux ». Il ajoute : “ si c'est là le sentiment et la voix générale de la nature, $M$. Descartes n'aura pas été le seul qui l'aura étouffée ; car je doute qu'il y en ait beaucoup d'autres que cet auteur, qui aient eu l'oreille aussi fine pour l'écouter ${ }^{11}$ n.

Arnauld s'attardera davantage sur la question de la distinction de l'âme et du corps, qu'il trouve plus importante que leur union.

La démonstration de la distinction réelle de l'âme et du corps, chez Descartes, avait déjà retenu l'attention de Arnauld quand il reçut de Mersenne le manuscrit des Méditations métaphysiques. Elle est au nombre des remarques qu'il adressa à Descartes et qui constituent les Quatrièmes objections. Elles provoquèrent de la part de Descartes une explication de la distinction de l'âme et du corps que Arnauld jugea entièrement satisfaisante. Aussi, dans sa réplique au doyen de Vitré, Arnauld ne manque-t-il pas de rappeler que tout le mérite de Descartes a été précisément d'avoir proposé une démonstration de la distinction réelle de l'âme et du corps. "Si il y a quelque chose ", écrit-il, a qui rende M. Descartes recommandable, c'est d'avoir si bien séparé notre âme d'avec notre corps, et d'avoir si bien établi que ce sont deux substances totalement distinctes, dont il n'y en a qu'une matérielle qu'on ne soit plus en peine, après cela, comment de voir

10. Cité par Arnauld dans Examen du Traité de l'essence du corps, O.C., tome 38, p. 138.

11. Ibid, p. 162. 
deux substances si différentes peuvent être unies pour ne faire qu'un seul homme ${ }^{12}$ s.

Arnauld, en effet, trouve plus important d'être persuadé de la distinction de l'âme et $\mathrm{du}$ corps que d'ignorer comment deux substances hétérogènes peuvent être unies. Il observe que les hommes sont assez convaincus de cette union. Chacun en fait l'expérience. Dieu a voulu que l'âme soit unie non seulement à son propre corps, mais par l'intermédiaire de son corps aux autres corps qui l'entomrent et qui peuvent avoir quelque rapport à sa propre conservation. Le recours à Dieu, c'est-à-dire à la toute-puissance divine, transforme en mystère ce qui pour l'entendement est une contradiction, un scandale. Il est en effet scandaleux, au regard de la logique, qu'un être composé de deux substances a complètes »l'une et l'autre soit en même temps une seule personne. L'imagination échoue à se représenter l'interaction du corps et de l'esprit immatériel. C'est une vérité de l'expérience et, comme le dit Descartes : "Chacun l'éprouve en soi même sans philosopher ».

Et lorsque le doyen de Vitré attribue à Descartes l'opinion des platoniciens selon laquelle l'âme est au corps ce qu'est un pilote à un vaisseau, métaphore qui illustre que l'union n'est pas substantielle, Arnauld réplique que l'accusation est injuste. Mais, à supposer même que ce fût là la pensée de Descartes, il estime a que ce serait une piqûre d'épingle en comparaison de l'importante plaie qu'il guérit en ruinant, par cette distinction, le sentiment impie de la mortalité de l'âme, qui n'est principalement fondée que sur ce qu'on la croit de même nature que le corps et qui est la plus damnable de toutes les erreurs ; ceux qui en sont prévenus étant portés à s'abandonner à toutes leurs passions parce qu'étant persuadés qu'il n'y a plus rien à

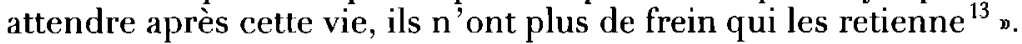

Quant à l'assimilation du corps à une machine, Arnauld se demande ce " qu'a donc de si mauvais le mot de machine et de si indigne de l'homme? "Il faut s'entendre sur les termes. Le mot machine signifie d'abord, explique-t-il à son adversaire, les ouvrages de l'art dont les pièces sont disposées et arrangées avec tant d'industrie pour produire un effet qui peut être admirable et surprenant, comme les automates, ou même utile, comme les montres. Les ouvrages de Dieu contiennent infiniment plus d'artifices que les ouvrages des hommes. Cependant, rien n'empêche qu'on leur applique ce terme qui enferme principalement dans sa notion la sagesse et l'industrie de l'ouvrier. Or, entre tous les ouvrages de Dieu qui composent notre monde créé, il n'y en a point de plus merveilleux et en quoi la sagesse de Dieu reluise davantage que les corps animés. Ce que signifie donc le mot machine appliqué au corps animé ou “organisé », ce sont " ces merveilleux

12. Ibid., p. 138 .

13. Ibid., p. 139. 
arrangements d'une infinité de parties dont la variété et la structure passent toute admiration ". Il n'y a donc aucune raison de reprocher à Descartes de mettre le corps de l'homme au rang des choses inanimées en l'identifiant à une machine.

L'explication cartésienne satisfait le bon sens et fait plus admirer la toute-puissance de Dieu.

Le problème sera de nouveau examiné dans le Discours de la Méthode. Au chapitre $\mathrm{V}$ du Discours, après avoir résumé ses analyses déjà exposées dans le Traité de l'homme, Descartes fait remarquer, à propos des automates, qu'il est possible, et l'industrie des hommes en est la preuve, de fabriquer une machine très perfectionnée qui ressemble en tous points à un animal sans qu'on puisse distinguer lequel est le a robot" ou l'animal vrai. Mais s'agissant des hommes, si l'on fabriquait des robots qui « eussent la ressemblance de nos corps et imitassent autant nos actions que moralement il serait possible, nous aurions toujours deux moyens très certains de reconnaître qu'elles ne seraient point pour autant de vrais hommes ${ }^{14}{ }^{\mathrm{n}}$.

C est par l'utilisation de signes linguistiques que l'on reconnaît les vrais hommes. Le langage a donc pour fonction d'identifier un vrai homme. En effet, on ne voit pas les animaux capables de composer des signes pour les faire servir en toutes sortes de rencontres. Ils peuvent bien imiter les sons, ils n'imitent pas la parole. Ils nous manifestent, certes, leurs passions, mais non leurs pensées. Il n'y a rien dans leurs cris, leurs gestes qui ne puisse se rapporter à une impulsion naturelle, mécanique. La différence entre le pseudo-langage animal et le vrai langage humain tient à la possibilité de dépasser les contacts corporels. La mécanique corporelle est purement spatiale, alors que la pensée de l'homme est vision, intuition.

Descartes en conclut que la faculté de la parole relève de la seule raison et qu'elle suppose l'existence d'une substance spirituelle, d'une âme immatérielle. Il va même jusqu'à déclarer que I 'on ne peut pas ramener au langage animal le langage des fous. Le langage des fous reste encore dans la sphère de l'humain. Le fou cartésien est un fou qui parle, qui pense, qui raisonne. Il manifeste par la forme de son discours qu'il est détenteur de la raison, même si, on peut le déplorer, il ne la suit pas.

Descartes prend quelque distance par rapport à l'opinion platonicienne selon laquelle l'âme serait logée dans le corps comme un pilote dans son navire. "Il ne suffit pas qu' elle soit logée dans le corps humain, ainsi qu'un pilote dans son navire, sinon peut-être pour mouvoir ses membres, mais qu'il est besoin qu'elle soit jointe et unie plus étroitement avec lui pour avoir, outre cela, des sentiments et des appétits semblables aux nôtres, et ainsi composer un vrai homme ». C'est donc par l'union substantielle qu'il convient de

14. Descartes, Discours de la Méthode, V, Garnier, tome 1, p. 628-629. 
définir le vrai homme. L'évocation de l'opinion platonicienne de l'âme logée dans le corps comme le pilote dans son navire est un rappel du dualisme platonicien que les philosophes de l'École rejetaient comme incapable à leurs yeux de rendre compte de l'unité de l'homme. Ce n'est pas une substance, c'est donc un accident ou un mode. Mais quelle est la nature de ce mode ? Est-elle spirituelle ou corporelle ? Comment, dès lors qu' elle est spirituelle, comprendre qu'une qualité spirituelle soit la modification d'un corps ? Inversement, de l'hétérogénéité radicale des deux substances, l'union ne saurait dériver qu'accidentellement et de façon inintelligible. L'expression de Regius définissant l'homme comme un "être par accident » traduit une pensée scolastique. Aussi, Descartes a-t-il refusé le mot de Regius. Selon Descartes, l'union est aussi naturelle que la distinction.

En conclusion, que nous apprend cette polémique? Rien de nouveau sur l'engagement cartésien de Arnauld. Elle confirme, au contraire, son caractère qui le pousse à réagir chaque fois que l'É glise catholique est attaquée. Dans cette affaire, c'est la croyance en l'immortalité de l'âme que menace le doyen de Vitré quand il s'attaque à la philosophie de Descartes. Aux yeux de ses contemporains, il convient de noter que Descartes a chassé pour quelque temps le doute et le fidéisme de la génération précédente, et renouvelé l'espérance chrétienne. Arnauld lui-même n'hésite pas à présenter Descartes, dans la réponse qu'il adresse à Le Moine, comme un envoyé de la Providence ayant pour mission de réduire les assauts des libertins et freiner la montée du matérialisme épicurien. Nicole sera de son sentiment :

On avait philosophé trois mille ans sur divers principes et il s'élève dans un coin de la terre un homme qui change toute la face cle la philosophie et qui prétend faire voir que tous ceux qui sont venus avant lui n'ont rien entendu dans les principes de la nature. Et ce ne sont pas seulement de vaines promesses car il faut avouer que le nouveau venu donne plus de lumière sur la connaissance des choses naturelles que tous les autres ensemble n'en avaient donné ${ }^{15}$.

Autrement dit, dans l'explication de la nature de l'âme, Nicole préfère Descartes à Lucrèce et à Épicure dont la philosophie dominante, en cette seconde moitié du XVII ${ }^{e}$ siècle, conduit à l'irreligion. La question de la spiritualité de l'âme est liée au problème de la destinée de l'homme. L'immortalité de l'âme était une préoccupation des contemporains. Même Bossuet se laissera conquérir par cette philosophie nouvelle avant de regretter, avec raison, que les imprudences des cartésiens fissent perdre à l’Église « le fruit qu'elle en pouvait espérer pour établir, dans l'esprit des philosophes, la divinité et la mortalité de l'âme ${ }^{16}$ m.

15. P. Nicole, Essais de Morale, de la faiblesse de l'homme, tome 1, p. 30-31.

16. Bossuet, Correspondance, citée par Sainte-Beuve, Port-Royal, Livre VI, éd. 1901, p. 367. 
La controverse qui oppose Arnauld et le doyen de Vitré a cependant le mérite de montrer une nouvelle vision chrétienne de l'union substantielle de l'âme et du corps. Par l'Incarnation et la théologie de la grâce, le corps acquiert une positivité qu'il n'avait pas chez Platon. Ce qui rend possible l'union intime avec l'âme, c'est la revalorisation du sensible que le christianisme reconnaît. Ainsi, en expliquant l'union réelle par notre affectivité, Descartes s'inscrit dans une vision chrétienne. Le corps, autant que l'âme, a sa part au salut. Dieu récompense et punit les hommes selon l'âme et selon le corps. L'usage des sens qui ne peut être sans la fonction de l'une et des deux parties dont nous sommes composés, contribue à la bonne ou à la mauvaise vie, aux désordres spirituels qui entraînent la damnation. Le christianisme renverse la vision païenne du corps en reconnaissant à ce corps sa participation à la spiritualité de l'âme. Le corps cesse d'être affecté du signe négatif. Les controverses théologiques sur la grâce au XVII ${ }^{e}$ siècle sont en effet le signe de cette nouvelle vision.

Il nous faut revenir sur l'un des arguments du doyen de Vitré. C' est celui qu'il oppose à Descartes en indiquant que sa doctrine du corps-machine est contradictoire avec la résurrection des corps. “ Dieu, rappelle-t-il, ne ressuscite pas des machines. " Arnauld reste persuadé que la philosophie de Descartes est avantageuse à la religion. Le Moine ne partage pas cet optimisme. Il craint, au contraire, que cette transparence, sous le contrôle des mathématiques, que Descartes veut étendre dans tous les domaines, peut être préjudiciable à la religion. La foi a, en effet, besoin de mystère. C'est la dimension du mystère qu'il ne retrouve pas dans la doctrine du corps-machine. Face au cartésianisme triomphant, celui d'Arnauld, on peut avoir de la sympathie pour quelqu'un qui veut redonner le sens de l'humain au corps, seul moyen de ne pas le considérer comme un instrument ou une machine.

Département de philosophie

Université de Dakar 